\title{
Difficulty in the identification and differentiation of clinically relevant Ochrobactrum species
}

The genus Ochrobactrum currently comprises nine species, Ochrobactrum anthropi (Holmes et al., 1988), Ochrobactrum intermedium (Velasco et al., 1998), Ochrobactrum lupini (Trujillo et al., 2005), Ochrobactrum tritici and Ochrobactrum grignonense (Lebuhn et al., 2006), Ochrobactrum gallinifaecis

(Kämpfer et al., 2003), Ochrobactrum oryzae (Tripathi et al., 2006), Ochrobactrum pseudintermedium (Teyssier et al., 2007) and Ochrobactrum cytisi (Zurdo-Piñeiro et al., 2007). Only three species, O. anthropi, O. intermedium and $O$. pseudintermedium, have been reported to occur in clinical material (Alnor et al., 1994; Appelbaum \& Campbell, 1980; Barson et al., 1987; Braun et al., 1996; Brivet et al., 1993; Cieslak et al., 1992, 1996; Earhart et al., 1997; Greven \& Nelson, 2001; Inoue et al., 1999; Jelveh \& Cunha, 1999; Kettaneh et al., 2003;

Mahmood et al., 2000; Perez-Blanco et al., 2005; Romero-Gomez et al., 2004; Vaidya et al., 2006; Wheen et al., 2002; Teyssier et al., 2007).

O. anthropi was described by Holmes et al. (1988) to accommodate oxidase-positive, Gram-negative, non-fermentative bacilli previously known as 'Achromobacter group Vd', occasionally infecting humans. Today O. anthropi is recognized as an emerging pathogen of low virulence, predominantly isolated from immunocompromised patients, but there are also 19 cases of it being isolated from immunocompetent hosts (Vaidya et al., 2006). Only two cases of $O$. intermedium infection have been reported. In 1999, O. intermedium was isolated from blood and a liver abscess in a patient after liver transplantation (Möller et al., 1999), and in 2005, a presumptive systemic $O$. intermedium infection in a patient with bladder cancer was reported (Apisarnthanarak et al., 2005). Recently a

The DDBJ/EMBL/GenBank accession number for the 16S rRNA gene sequence of isolate RMA 16449 is AM409326. novel species, O. pseudintermedium, was described that was isolated from patients hospitalized in intensive-care units (Teyssier et al., 2007). The differentiation of Ochrobactrum species by physiological tests is quite difficult, because of their high phenotypic similarity (Table 1). $O$. anthropi is often the only Ochrobactrum species present in the databases of commercially available identification systems (including the API 20NE kit) (Teyssier et al., 2005a) and as a consequence $O$. intermedium may be misidentified as O. anthropi as occurred in a recently published case report describing O. anthropi as the causative agent of a pelvic abscess in an immunocompetent host (Vaidya et al., 2006). The initial identification of that isolate (RMA 16449) as O. anthropi (Vaidya et al., 2006) was performed with the API 20E kit (bioMérieux) and by partial 16S rRNA gene sequencing. Strain RMA 16449 was later included in comparative analysis to study the genetic diversity among 112 Ochrobactrum strains by applying a polyphasic approach using extended 16S rRNA gene analysis, $r e c A$ gene sequencing, fatty acid analysis and DNA-DNA hybridization studies. Of the 16S rRNA gene, 1390 bp were sequenced as described by Kämpfer et al. (2003). In the BLASTN search using the sequence information from the entire 1390 bp fragment (GenBank accession no. AM409326), the highest similarity of $100 \%$ was indeed detected with ' $O$. anthropi' strain CCUG 39736 (GenBank accession no.

AM114408). However, to add to the confusion, this reference strain, initially identified as $O$. anthropi, was recently transferred to O. intermedium (see the CCUG Homepage: www.ccug.se). An identity of $99 \%$ was detected with the type strain of O. intermedium, CCUG $24694^{\mathrm{T}}$ (GenBank accession no. AM114411), and only $97.8 \%$ to the type strain of $O$. anthropi, LMG $3331^{\mathrm{T}}$ (GenBank accession no. AM114398). Hence isolate RMA 16994 appeared to be more closely related to $O$. intermedium than to O. anthropi.

Identification of isolate RMA 16994 as $O$. intermedium was confirmed by the presence of the $O$. intermedium-specific sequence motifs ggctaat and ggttagtggagacactatcc at positions nt 487493 and nt 905-924 of the 1390 bp 16S rRNA gene fragment as described by Scholz et al. (2006). The second motif is also shared with Brucella spp. However, both motifs are absent in O. anthropi and are instead represented by the sequences gactttt and ggacacagagatgtgtct at these positions, respectively.

Using $r e c A$ as a highly discriminatory marker to study the phylogeny of Ochrobactrum spp. at the inter- and intraspecies level (Scholz et al., 2006) confirmed isolate RMA 16449 as $O$. intermedium (results not shown).

Fatty acid analysis using the Sherlock System (MIDI; Kämpfer \& Kroppenstedt, 1996) and comparison with the Sherlock database provided the identification result O. anthropi with an ID score of 0.556 , indicating that fatty acid analysis is not sufficient for differentiation at the species level (results not shown). Selected results of the physiological characterization (according to Kämpfer et al., 1991) compared with those from O. anthropi CIP $14970^{\mathrm{T}}$ and $O$. intermedium LMG $3301^{\mathrm{T}}$ are given in Table 1. Only very few biochemical tests are able to differentiate these species. For final species confirmation, DNA-DNA hybridization experiments were performed with RMA 16449 and O. intermedium LMG $3301^{\mathrm{T}}$ using the method described by Ziemke et al. (1998) modified as described by Kämpfer et al. (2003). Strain RMA 16449 showed $79 \%$ DNA-DNA similarity (mean value of two hybridizations) to $O$. intermedium LMG $3301^{\mathrm{T}}$, confirming that RMA 16449 is member of this species, since the homology demonstrated was $>70 \%$. 
Table 1. Physiological characteristics of selected type strains of Ochrobactrum species tested with the methods according to Kämpfer et al. (1991)

Taxa: 1, O. anthropi CIP $14970^{\mathrm{T}} ; 2, O$. intermedium LMG $3301^{\mathrm{T}}$; 3, O. intermedium RMA 16449. +, Positive; -, negative; $(+)$, weakly positive. All strains were positive for hydrolysis of L-alanine $p \mathrm{NA}^{*}$ and L-proline $p \mathrm{NA} \ddagger$ and weak hydrolysis of bis- $p \mathrm{NP}^{\star}$ phosphate and L-glutamate- $\gamma$-3-carboxy$p$ NA. All strains were negative for hydrolysis of aesculin $\dagger, p \mathrm{NP} \beta$-D-galactopyranoside $\dagger$, $p \mathrm{NP} \beta$-D-glucuronide, $p \mathrm{NP} \alpha$-D-glucopyranoside, $p \mathrm{NP} \beta$-D-glucopyranoside, $p \mathrm{NP}$ phenyl phosphonate, $p \mathrm{NP}$ phosphorylcholine and 2-deoxythymidine- $5^{\prime}$ - $p \mathrm{NP}$ phosphate. All strains were also positive for assimilation of L-arabinose $\dagger$, D-cellobiose $\dagger$, D-galactose $\dagger$, gluconate, D-glucose, D-mannose, Dmaltose $\dagger \neq, \quad N$-acetyl-D-glucosamine $\dagger, \quad N$ acetyl-D-galactosamine, D-ribose $\dagger, \mathrm{D}$-xylose, D-fructose, adonitol $\dagger, \quad$ i-inositol $\dagger, \quad$ Lrhamnose $\dagger, \quad \mathrm{D}$-sorbitol $\dagger$, cis-aconitate, citrate $\dagger$, DL-3-hydroxybutyrate $\dagger$, acetate, 4 -aminobutyrate, $\beta$-alaninet, propionate $\dagger$, fumarate $\dagger$, glutarate, DL-lactate, L-malate $\dagger$, oxoglutarate $\dagger$, pyruvate $\dagger$, L-alanine, Laspartate $\dagger, \mathrm{L}$-histidine $\dagger$, L-leucine $\dagger, \mathrm{L}$-proline, L-serine $\dagger$, ornithine and $\uparrow 4$-hydroxybenzoate. All strains were negative for assimilation of $p$-arbutin, salicin, suberate $\dagger$, putrescine, Lphenylalanine, L-tryptophan, 3-hydroxybenzoate, adipate $\neq$, itaconate, mesaconate, phenylacetate $\dagger, \alpha$-D-melibiose $\dagger$ and azelate.

\begin{tabular}{|lccc|}
\hline Test & $\mathbf{1}$ & $\mathbf{2}$ & $\mathbf{3}$ \\
\hline Susceptibility to: & & & \\
$\quad$ Colistin, polymyxin & + & - & - \\
Assimilation of: & & & \\
$\quad$ Sucrose $\dagger \neq, \mathrm{D}-\mathrm{trehalose}$ & + & - & - \\
Maltitol & + & - & - \\
trans-Aconitate $\dagger$ & - & $(+)$ & - \\
\hline
\end{tabular}

${ }^{*} p \mathrm{NP}$, para-nitrophenyl; $p \mathrm{NA}$, para-nitroanilide.

$\dagger$ Test (based on a different method) was also performed by Holmes et al. (1988) with $O$. anthropi and gave congruent results.

$\ddagger$ Test (based on a different method) was also performed by Velasco et al. (1998) with O. intermedium and gave congruent results.

The majority of Ochrobactrum infections have been attributed to $O$. anthropi whereas only two cases of $O$. intermedium infection, one in a patient with bladder cancer (Apisarnthanarak et al., 2005) and another case after liver transplantation (Möller et al., 1999), have been reported. In both cases, the authors emphasized that correct identification and differentiation of $O$. anthropi and O. intermedium by biochemical test systems that are routinely used in clinical diagnosis is difficult and even impossible since $O$. intermedium is not present in the databases of these test systems. Furthermore, misidentification of Brucella as O. anthropi using API 20NE has been reported (Elsaghir \& James, 2003).

This finding is of particular clinical importance since correct identification is the prerequisite for applying an adequate therapy. In their article, Elsaghir \& James (2003) reviewed the literature on $O$. anthropi infections of which in 32 cases $O$. anthropi was identified by API 20NE. Some of these cases presented with a clinical picture similar to brucellosis requiring multiple antibiotics for therapy. The authors therefore speculated that at least some of these cases might have been caused by Brucella that had been misidentified as $O$. anthropi.

At present, 16S rRNA gene sequencing is used for the identification and differentiation of $O$. anthropi, $O$. intermedium and Brucella spp. However, this approach, in particular partial sequencing, is prone to lead to misidentification of these pathogens, because of high sequence similarities of $>98 \%$. As an example, in the case of the $O$. intermedium infection described by Apisarnthanarak et al. (2005), 16S rRNA gene sequencing was not suitable for identifying the disease-causing agent as $O$. intermedium since sequence similarities of $99 \%$ were obtained with both O. anthropi and $O$. intermedium. The high similarity to O. anthropi reported in this article might have resulted from sequences of misidentified strains deposited in the EMBL database, as described above for $O$. anthropi CCUG 39736. We therefore suggest that the obtained BLAST results are carefully checked for their accuracy by the presence of $O$. anthropi- or $O$. intermedium-specific sequence motifs as described by Scholz et al. (2006). Vaidya et al. (2006) identified ' $O$. anthropi' using biochemical testing and partial sequencing of the $16 \mathrm{~S}$ rRNA gene that we clearly identified in this study as O. intermedium using extended 16S rRNA and recA gene analysis as well as DNA-DNAhybridization studies, emphasizing the need for additional molecular typing approaches in order to correctly identify O. anthropi and O. intermedium.

In this context, $\operatorname{rec} A$ analysis was shown to serve (Scholz et al., 2006) as a suitable tool for differentiating O. anthropi, $O$. intermedium and Brucella species. We therefore recommend $r e c A$ gene analysis for accurate typing of these organisms. RecA can also be used to correctly identify O. pseudintermedium (H. C. Scholz, personal communication), which was recently isolated from clinical specimens.

In addition to molecular typing, colistin (polymyxin E) and polymyxin B are recommended for differentiating between O. anthropi and O. intermedium in routine diagnosis (Velasco et al., 1998; Teyssier et al., 2005a), whereby O. anthropi is sensitive and $O$. intermedium is resistant to both antibiotics. However, in a screening assay, 5 out of 65 confirmed O. anthropi strains were resistant and 3 out of 17 tested $O$. intermedium strains were sensitive to both antibiotics (H. C. Scholz, personal communication).

In summary, commercially available test systems used in routine diagnosis are not suitable for species discrimination within the genus Ochrobactrum. We therefore recommend molecular typing (Lebuhn et al., 2006; Scholz et al., 2006; Teyssier et al., 2003a, b, 2005a, b) in addition to physiological testing for a precise species identification.

Besides sequencing of the $16 \mathrm{~S}$ rRNA gene, analysis of recA (Scholz et al., 2006) allows accurate identification and differentiation of $O$. anthropi, O. intermedium and $O$. pseudintermedium. However, there is still a need to develop differentiating PCR assays for the rapid and correct identification of these organisms.

\section{Peter Kämpfer, ${ }^{1}$ Diane M. Citron, ${ }^{2}$ Ellie J. C. Goldstein ${ }^{3}$ and Holger C. Scholz ${ }^{4}$}

\footnotetext{
${ }^{1}$ Institut für Angewandte Mikrobiologie, Justus-Liebig Universität Giessen, D-35392 Giessen, Germany
} 
${ }^{2}$ R. M. Alden Research Laboratory, Santa Monica, CA 90404, USA

${ }^{3}$ David Geffen School of Medicine at Los Angeles, Los Angeles, CA 90073, USA

${ }^{4}$ Bundeswehr Institute of Microbiology, D-80937 Munich, Germany

Correspondence: Peter Kämpfer (peter.kaempfer@agrar.uni-giessen.de)

Alnor, D., Frimodt-Moller, N., Espersen, F. \& Frederiksen, W. (1994). Infections with the unusual human pathogens Agrobacterium species and Ochrobactrum anthropi. Clin Infect Dis 18, 914-920.

Apisarnthanarak, A., Kiratisin, P. \& Mundy, L. M. (2005). Evaluation of Ochrobactrum intermedium bacteremia in a patient with bladder cancer. Diagn Microbiol Infect Dis 53, 153-155.

Appelbaum, P. C. \& Campbell, D. B. (1980).

Pancreatic abscess associated with Achromobacter group Vd biovar 1. J Clin Microbiol 12, 282-283.

Barson, W. J., Cromer, B. A. \& Marcon, M. J. (1987). Puncture wound osteochondritis of the foot caused by CDC group Vd. J Clin Microbiol 25, 2014-2016.

Braun, M., Jonas, J. B., Schonherr, U. \& Naumann, G. O. (1996). Ochrobactrum anthropi endophthalmitis after uncomplicated cataract surgery. Am J Ophthalmol 122, 272-273.

Brivet, F., Guibert, M., Kiredjian, M. \& Dormont, J. (1993). Necrotizing fasciitis, bacteremia, and multiorgan failure caused by Ochrobactrum anthropi. Clin Infect Dis 17, 516-518.

Cieslak, T. J., Robb, M. L., Drabick, C. J. \& Fischer, G. W. (1992). Catheter-associated sepsis caused by Ochrobactrum anthropi: report of a case and review of related nonfermentative bacteria. Clin Infect Dis 14, 902-907.

Cieslak, T. J., Drabick, C. J. \& Robb, M. L. (1996). Pyogenic infections due to Ochrobactrum anthropi. Clin Infect Dis 22, 845847.

Earhart, K. C., Boyce, K., Bone, W. D. \& Wallace, M. R. (1997). Ochrobactrum anthropi infection of retained pacemaker leads. Clin Infect Dis 24, 281-282.

Elsaghir, A. A. \& James, E. A. (2003).

Misidentification of Brucella melitensis as Ochrobactrum anthropi by API 20NE. J Med Microbiol 52, 441-442.

Greven, C. M. \& Nelson, K. C. (2001). Chronic postoperative endophthalmitis secondary to Ochrobactrum anthropi. Retina 21, 279-280.

Holmes, B., Popoff, M., Kiredjian, M. \& Kersters, K. (1988). Ochrobactrum anthropi gen. nov., sp. nov. from human clinical specimens and previously known as group Vd. Int J Syst Bacteriol 38, 406-416.
Inoue, K., Numaga, J., Nagata, Y., Sakurai, M., Aso, N. \& Fujino, Y. (1999). Ochrobactrum anthropi endophthalmitis after vitreous surgery. Br J Ophthalmol 83, 502.

Jelveh, N. \& Cunha, B. A. (1999). Ochrobactrum anthropi bacteremia. Heart Lung 28, 145-146.

Kämpfer, P. \& Kroppenstedt, R. M. (1996). Numerical analysis of fatty acid patterns of coryneform bacteria and related taxa. Can J Microbiol 42, 989-1005.

Kämpfer, P., Steiof, M. \& Dott, W. (1991). Microbiological characterization of a fuel-oil contaminated site including numerical identification of heterotrophic water and soil bacteria. Microb Ecol 21, 227-251.

Kämpfer, P., Buczolits, S., Albrecht, A., Busse, H.-J. \& Stackebrandt, E. (2003). Towards a standardized format for the description of a novel species (of an established genus): Ochrobactrum gallinifaecis sp. nov. Int J Syst Evol Microbiol 53, 893-896.

Kettaneh, A., Weill, F. X., Poilane, I., Fain, O., Thomas, M., Herrmann, J. L. \& Hocqueloux, L. (2003). Septic shock caused by Ochrobactrum anthropi in an otherwise healthy host. J Clin Microbiol 41, 1339-1341.

Lebuhn, M., Bathe, S., Achouak, W., Hartmann, A., Heulin, T. \& Schloter, M. (2006).

Comparative sequence analysis of the internal transcribed spacer 1 of Ochrobactrum species. Syst Appl Microbiol 29, 265-275.

Mahmood, M. S., Sarwari, A. R., Khan, M. A., Sophie, Z., Khan, E. \& Sami, S. (2000). Infective endocarditis and septic embolization with Ochrobactrum anthropi: case report and review of literature. J Infect 40, 287-290.

Möller, L. V. M., Arends, J. P., Hermsen, H. J. M. Talens, A., Terpstra, P. \& Slooff, M. J. H. (1999). Ochrobactrum intermedium infection after liver transplantation. J Clin Microbiol 37, 241-244.

Perez-Blanco, V., Gacia-Caballero, J., Dominguez-Melcon, F. J. \& Gomez-Limon, I. M. (2005). Ochrobactrum anthropi infectious endocarditis in an immunocompetent patient. Enferm Infecc Microbiol Clin 23, 111-112.

Romero Gomez, M. P., Peinado Esteban, A. M., Sobrino Daza, J. A., Saez Nieto, J. A., Alvarez, D. \& Pena Garcia, P. (2004). Prosthetic mitral valve endocarditis due to Ochrobactrum anthropi: case report. J Clin Microbiol 42, 33713373.

Scholz, H. C., Tomaso, H., Dahouk, S. A., Witte, A., Schloter, M. \& Kämpfer, P. (2006). Genotyping of Ochrobactrum anthropi by recAbased comparative sequence, PCR-RFLP, and $16 \mathrm{~S}$ rRNA gene analysis. FEMS Microbiol Lett 257, 7-16.

Teyssier, C., Jumas-Bilak, E., Marchandin, H., Jean-Pierre, H., Jeannot, J. L., Dusart, G., Foulongne, V. \& de Buochberg, S. M. (2003a). Species identification and molecular epidemiology of bacteria belonging to Ochrobactrum genus. Pathol Biol 51, 5-12.
Teyssier, C., Marchandin, H., de Buochberg, M. S., Ramuz, M. \& Jumas-Bilak, E. (2003b). Atypical 16S rRNA gene copies in Ochrobactrum intermedium strains reveal a large genomic rearrangement by recombination between rrn copies. J Bacteriol 185, 2901-2909.

Teyssier, C., Marchandin, H., Jean-Pierre, H., Diego, I., Darbas, H., Jeannot, J. L., Gouby, A. \& Jumas-Bilak, E. (2005a). Molecular and phenotypic features for identification of the opportunistic pathogens Ochrobactrum spp. J Med Microbiol 54, 945-953.

Teyssier, C., Marchandin, H., Masnou, A., Jeannot, J. L., de Buochberg, M. S. \& Jumas-Bilak, E. (2005b). Pulsed-field gel electrophoresis to study the diversity of wholegenome organization in the genus Ochrobactrum. Electrophoresis 26, 2898-2907.

Teyssier, C., Marchandin, H., Jean-Pierre, H., Masnou, A., Dusart, G. \& Jumas-Bilak, E. (2007). Ochrobactrum pseudintermedium sp. nov., a novel member of the family Brucellaceae, isolated from human clinical samples. Int J Syst Evol Microbiol 57, 1007-1013.

Tripathi, A. K., Verma, S. C., Chowdhury, S. P., Lebuhn, M., Gattinger, A. \& Schloter, M. (2006) Ochrobactrum oryzae sp. nov., an endophytic bacterial species isolated from deep-water rice in India. Int J Syst Evol Microbiol 56, 1677-1680.

Trujillo, M. E., Willems, A., Abril, A., Planchuelo, A. M., Rivas, R., Ludeña, D., Mateos, P. F., Martínez-Molina, E. \& Velázquez, E. (2005). Nodulation of Lupinus by strains of the new species Ochrobactrum lupini sp. nov. Appl Environ Microbiol 71, 1318-1327.

Vaidya, S. A., Citron, D. M., Fine, M. B. Murakami, G. \& Goldstein, E. J. C. (2006). Pelvic abscess due to Ochrobactrum anthropi in an immunocompetent host: case report and review of the literature. J Clin Microbiol 44, 1184-1186.

Velasco, J., Romero, C., López-Goñi, I., Leiva, J., Diaz, R. \& Moriyón, I. (1998). Evaluation of the relatedness of Brucella spp. and Ochrobactrum anthropi and description of Ochrobactrum intermedium sp. nov., a new species with a closer relationship to Brucella spp. Int J Syst Bacteriol 48, 759-768.

Wheen, L., Taylor, S. \& Godfrey, K. (2002). Vertebral osteomyelitis due to Ochrobactrum anthropi. Intern Med J 32, 426-428.

Ziemke, F., Höfle, M., Lalucat, J. \& RossellóMora, R. (1998). Reclassification of Shewanella putrefaciens Owen's genomic group II as Shewanella baltica sp. nov. Int J Syst Bacteriol $\mathbf{4 8}$ 179-186.

Zurdo-Piñeiro, J. L., Rivas, R., Trujillo, M. E., Vizcaíno, N., Carrasco, J. A., Chamber, M., Palomares, A., Mateos, P. F., Martínez-Molina, E. \& Velázquez, E. (2007). Ochrobactrum cytisi sp. nov., isolated from nodules of Cytisus scoparius in Spain. Int J Syst Evol Microbiol 57, 784-788. 